\title{
Quark mass dependence of hyperonic interactions from lattice QCD
}

\author{
K. Sasaki* \\ Center of Computational Sciences, University of Tsukuba \\ Tsukuba, Ibaraki 305-8577, Japan \\ E-mail: kenjis@het.ph.tsukuba.ac.jp \\ for HAL QCD collaboration

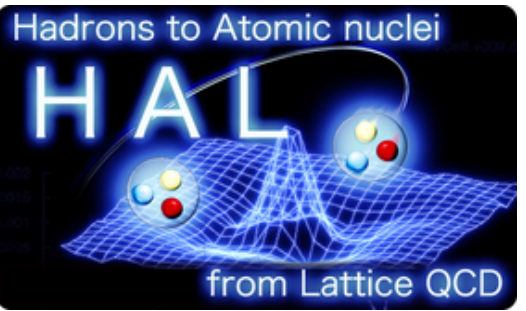

\begin{abstract}
We studied hyperonic interactions by lattice QCD simulation throuth coupled channel formalism. Our approach to baryon-baryon interactions is deriving a potential from inverting Schrödinger equation using Nambu-Bethe-Salpeter (NBS) wave function simulated on the lattice. The quark mass dependences and flavor SU(3) breaking effects of the potential matrix are also discussed by comparing with results of gauge configurations with different quark masses. Our numerical results are obtained from three ensembles of 2+1 flavor QCD gauge configurations, which corresponds to $m_{\pi} \sim 700,570$ and $410 \mathrm{MeV}$, provided by the PACS-CS Collaboration.
\end{abstract}

The 30 International Symposium on Lattice Field Theory - Lattice 2012,

June 24-29, 2012

Cairns, Australia

\footnotetext{
* Speaker.
} 


\section{Introduction}

Investigations of the baryon-baryon $(B B)$ interactions are truly crucial for the deeper understanding of atomic nuclei, structure of neutron stars and supernova explosions. So far, it has not been succeeded to deduce nuclear force from fundamental theory, quantum chromo-dynamics (QCD). A series of investigations by HAL QCD collaboration [1, 2, 3, 4, 5, 6, 7, 9,8 changes this situation and enables us to determine $B B$ potentials from lattice QCD Since potentials obtained in our method are faithful to the scattering phase shifts, the method would potentially be a complement to baryon-baryon scattering experiments.

An extension of the HAL QCD method to the coupled channel formalism is indispensable to investigate a two-baryon systems with strangeness. Especially for the case of strangeness $S=-2$ system with isospin $I=0$, energy levels of $\Lambda \Lambda, N \Xi$ and $\Sigma \Sigma$ are very close and a ground state saturation is not expected. The extension could enable us to explore the $B B$ potential in such system and then allow us to see the fate of the H-dibaryon at the physical quark masses with the (large) SU(3) breaking. It would also give us a chance to reveal the roles of quarks in baryons based on QCD because the short range repulsion in nuclear force are originated by the quark Pauli effect in two baryons [10].

Using the potentials derived from our method, it is interesting to seek a possibility for an existence of a bound state in two-baryon systems other than the deutron, especially in strange sector. In the flavor SU(3) symmetric world simulated by lattice QCD, the investigation by our method indicates an existence of the bound state in the flavor singlet channel with strangeness $S=-2$ [11]12], which correponds to the H-dibaryon state, predicted by R. L. Jaffe [13]. Even if the small SU(3) breaking effect is introduced, by applying the conventional Lüscher formula, it is shown that the $H$-dibaryon state exists with the binding energy of about $14 \mathrm{MeV}$ at $m_{\pi} \sim 389 \mathrm{MeV}$ [14].

The paper is organized as follows. First, We briefly show the extension procedure of HAL QCD method to the coupled channel formalism in Sect. 2. Setups of our numerical simulations are given in Sect. 3. The results of hyperonic potentials are presented in Sect. 4 Conclusions and outlook are given in Sect. 5.

\section{Coupled channel formalism}

The (equal time) NBS wave function is defined with local composite operators for a baryon, $B(\vec{x})=\varepsilon_{a b c}\left(q_{a}^{T}(\vec{x}) C \gamma_{5} q_{b}(\vec{x})\right) q_{c}(\vec{x})$ omitting a flavor structures for simplicity, as

$$
\psi^{B_{1} B_{2}}(\vec{r}, E)=\sum_{\vec{x}}\left\langle 0\left|B_{1}(\vec{x}+\vec{r}) B_{2}(\vec{x})\right| E\right\rangle
$$

which is embeded in the $R$-correlator given by

$$
R_{\mathscr{I}}^{B_{1} B_{2}}(t, \vec{r})=\sum_{\vec{x}} \frac{\left\langle 0\left|B_{1}(t, \vec{x}+\vec{r}) B_{2}(t, \vec{x}) \overline{\mathscr{I}}(0)\right| 0\right\rangle}{e^{-\left(m_{1}+m_{2}\right) t}} \propto A_{E} \psi^{B_{1} B_{2}}(\vec{r}, E) e^{-\tilde{E} t}
$$

for a moderate value of $t$, where $A_{E}=\langle E|\overline{\mathscr{I}}(0)| 0\rangle$ and $\mathscr{I}(t)$ is a optimized source operator, which creates the eigenstate of the energy $E$ with a baryon number $B=2$. Assuming the flavors of 
quark as $u$ for "up", $d$ for "down" and $s$ for "strange", the flavor structure for baryons are defined as

$$
\begin{array}{ll}
(S=0, I=1 / 2) & : p=u d u \quad n=u d d \\
(S=1, I=1) & : \Sigma^{+}=-u s u \quad \Sigma^{0}=-(d s u+u s d) / \sqrt{2} \quad \Sigma^{-}=-d s d \\
(S=1, I=0) & : \Lambda=-(d s u+s u d-2 u d s) / \sqrt{6} \\
(S=2, I=1 / 2) & : \Xi^{0}=s u s \quad \Xi^{-}=s d s .
\end{array}
$$

Once the energy $E$ is determined, the asymptotic momentum $p$ in center-of-mass $(\mathrm{CM})$ frame is defined through

$$
E=\sqrt{m_{1}^{2}+p^{2}}+\sqrt{m_{2}^{2}+p^{2}}
$$

and the $\tilde{E}$ is defined as $\tilde{E} \equiv E-m_{1}-m_{2}$.

The most general form of the Schrödinger equation for wave function $\Psi(\vec{r}, E)$ is given by using a reduced mass $\mu$ and an asymptotic momentum $p$ in $\mathrm{CM}$ frame as,

$$
\left[\frac{p^{2}}{2 \mu}-H_{0}\right] \Psi(\vec{r}, E)=\int d^{3} \vec{r}^{\prime} U\left(\vec{r}, \vec{r}^{\prime}\right) \Psi\left(\vec{r}^{\prime}, E\right)
$$

where $H_{0}$ is the free Hamiltonian, $U\left(\vec{r}, \vec{r}^{\prime}\right)$ is an energy independent non-local potential [2]. The derivative expansion is performed to handle the non-locality of potential as $U\left(\vec{r}, \vec{r}^{\prime}\right)=\left(V_{L O}+\right.$ $\left.V_{N L O}+\cdots\right) \delta\left(\vec{r}-\vec{r}^{\prime}\right)$, where $N^{n} L O$ term is of $O\left(\vec{\nabla}^{n}\right)$. At low energies, efficiency of derivative expansion was confirmed in Ref. [6].

Now we assume that the total wave function $\Psi(\vec{r}, E)$ contains two independent channels, denoted by $\alpha$ and $\beta$. The eq. (2.5) can be written as the coupled channel form of Schrödinger equation at the leading order of the derivative expansion for the non-local potential,

$$
\left(\begin{array}{c}
\left(\frac{p_{\alpha}^{2}}{2 \mu_{\alpha}}+\frac{\nabla^{2}}{2 \mu_{\alpha}}\right) \psi^{\alpha}(\vec{r}, E) \\
\left(\frac{p_{\beta}^{2}}{2 \mu_{\beta}}+\frac{\nabla^{2}}{2 \mu_{\beta}}\right) \psi^{\beta}(\vec{r}, E)
\end{array}\right)=\left(\begin{array}{c}
V_{\alpha}^{\alpha}(\vec{r}) V_{\beta}^{\alpha}(\vec{r}) \\
V_{\alpha}^{\beta}{ }_{\alpha}(\vec{r}) V_{\beta}^{\beta}{ }_{\beta}(\vec{r})
\end{array}\right)\left(\begin{array}{c}
\psi^{\alpha}(\vec{r}, E) \\
\psi^{\beta}(\vec{r}, E)
\end{array}\right) .
$$

Combining another coupled channel equation with different energy, we can derive the potential matrix by inverting the equation. This is an extension of the HAL QCD method to the coupled channel formalism [15].

Further improvement has been proposed in [16] that potential is extracted without assuming a single channel saturation. Using the non-relativistic expansion that $E-m_{1}-m_{2} \simeq p^{2} / 2 \mu$, we can easily obtain the kinetic energy term by the time derivative of the $R$-correlator as

$$
-\frac{\partial}{\partial t} R_{\mathscr{I}}^{B_{1} B_{2}}(t, \vec{r}) \simeq \frac{p^{2}}{2 \mu} A_{E} \psi^{B_{1} B_{2}}(\vec{r}, E) e^{-\left(E-m_{1}-m_{2}\right) t} .
$$

Taking into account the improvement for eq. (2.6), we therefore obtain the coupled channel Schrödinger equation given in terms of the $R$-correlators combining with different $\mathscr{I}$ 's (energies) as

$$
\begin{aligned}
& \left(\begin{array}{ll}
V_{\alpha}^{\alpha}(\vec{r}) & V^{\alpha}{ }_{\beta}(\vec{r}) x \\
V^{\beta}{ }_{\alpha}(\vec{r}) x^{-1} & V^{\beta}{ }_{\beta}(\vec{r})
\end{array}\right) \\
& =\left(\begin{array}{cc}
\left(\frac{\nabla^{2}}{2 \mu_{\alpha}}-\frac{\partial}{\partial t}\right) R_{\mathscr{I}_{1}}^{\alpha}(t, \vec{r}) & \left(\frac{\nabla^{2}}{2 \mu_{\beta}}-\frac{\partial}{\partial t}\right) R_{\mathscr{I}_{1}}^{\beta}(t, \vec{r}) \\
\left(\frac{\nabla^{2}}{2 \mu_{\alpha}}-\frac{\partial}{\partial t}\right) R_{\mathscr{I}_{2}}^{\alpha}(t, \vec{r}) & \left(\frac{\nabla^{2}}{2 \mu_{\beta}}-\frac{\partial}{\partial t}\right) R_{\mathscr{I}_{2}}^{\beta}(t, \vec{r})
\end{array}\right)\left(\begin{array}{c}
R_{\mathscr{I}_{1}}^{\alpha}(t, \vec{r}) R_{\mathscr{I}_{2}}^{\alpha}(t, \vec{r}) \\
R_{\mathscr{I}_{1}}^{\beta}(t, \vec{r}) R_{\mathscr{I}_{2}}^{\beta}(t, \vec{r})
\end{array}\right)^{-1}
\end{aligned}
$$

where $x \equiv \exp \left(-\left(m_{\beta_{1}}+m_{\beta_{2}}\right) t\right) / \exp \left(-\left(m_{\alpha_{1}}+m_{\alpha_{2}}\right) t\right)$. 
Table 1: Summary table of gauge ensembles and hadron masses in unit of MeV.

\begin{tabular}{lllllllll}
\hline & $\kappa_{u d}$ & $\kappa_{s}$ & $\pi$ & $K$ & $N$ & $\Lambda$ & $\Sigma$ & $\Xi$ \\
\hline Esb1 & 0.13700 & 0.13640 & $701(1)$ & $789(1)$ & $1585(5)$ & $1644(5)$ & $1660(4)$ & $1710(5)$ \\
Esb2 & 0.13727 & 0.13640 & $570(1)$ & $713(1)$ & $1411(12)$ & $1504(10)$ & $1531(11)$ & $1610(9)$ \\
Esb3 & 0.13754 & 0.13640 & $411(2)$ & $635(2)$ & $1215(12)$ & $1351(8)$ & $1400(10)$ & $1503(7)$ \\
\hline
\end{tabular}

\section{Numerical simulations}

In the calculation we employ $2+1$-flavor full QCD gauge configurations from Japan Lattice Data Grid(JLDG)/International Lattice Data Grid(ILDG) [17]. The PACS-CS Collaboration generated $L^{3} \times T=32^{3} \times 64$ lattice with a renormalization-group improved gauge action at $\beta=6 / g^{2}=$ 1.90 and a non-perturbatively $O(a)$ improved clover quark action with $C_{S W}=1.715$, corresponding to lattice spacings of $a=0.091 \mathrm{fm}\left(a^{-1}=2.176 \mathrm{GeV}\right)$ [18]. The spacial size of them is about $(2.9 \mathrm{fm})^{3}$ in physical unit. In order to investigate quark mass dependences of $B B$ interaction, three sets of hopping parameters for the $u$ and $d$ quark masses with fixed $s$-quark mass ( $\kappa_{s}=0.13640$ ) are considered named as Esb1, Esb2 and Esb3 respectively corresponging to $m_{\pi} \simeq 700,570$ and $410 \mathrm{MeV}$, given in Table 1

Quark propagators are calculated with the spatial wall source at $t=0$ with the Dirichlet boundary condition in temporal direction at $t=32+t_{0}$ which rules out an opposite propagation of two baryons in temporal direction. The wall source is placed at 16 different time slices on each of different gauge configuration ensembles, in order to enhance the signals, together with the average over forward and backward propagations in time. An average over the cubic group is taken for the sink operator, in order to obtain the S-wave in the $B B$ wave function.

\section{Results and discussions}

We consider the potential matrix in the ${ }^{1} S_{0}$ and $I=0$ channel, in which the $H$ dibaryon state appears if it exists. First, we look at the diagonal components of potential matrix shown in upper three panels of Fig. 1 All of them have a repulsive core at short distance. The strength of the repulsion in each channel, however, varies, reflecting properties of its main component in the irreducible representation of the flavor SU(3): The diagonal potential in the $\Sigma \Sigma$ channel, whose main component is the symmetric-octet in $\mathrm{SU}(3)$, is most repulsive, since the symmetric-octet has the strongest repulsion at all distances in the SU(3) limit [4] and this property holds even with the SU(3) breaking. On the other hand, diagonal potentials in $N \Xi$ and $\Lambda \Lambda$ channels has not only a repulsion at short distance but also an attraction at medium distance, due to a mixture between the repulsive symmetric-octet potential and the attractive flavor singlet potential [4]. We can see a growth of short range repulsion as decreasing of $u$ and $d$ quark masses but there are no clear enhancement at long distances.

Next, we see the off-diagonal components of potential matrix shown in lower three panels of Fig. 1 Comparing these three transition potentials, the $\Lambda \Lambda-N \Xi$ transition potential is smaller than other transition potentials. Therefore, the $N \Xi$ to $\Lambda \Lambda$ decay rate is expected to be relatively 

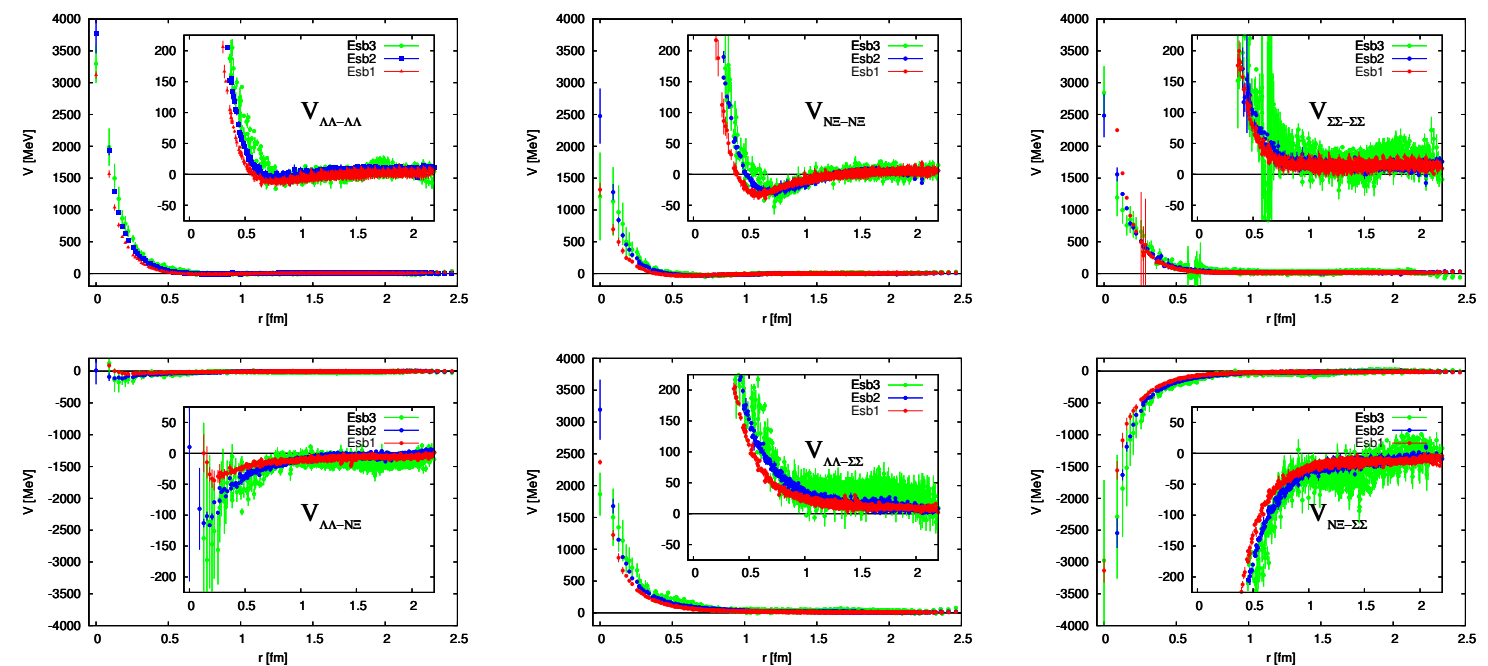

Figure 1: Potentials in ${ }^{1} S_{0}$ with $S=-2$ and $I=0$ in particle basis. Upper three panels are diagonal parts of potential matrix. Lower three panels are off-diagonal ones. Red, blue and green symbols stand for the result with Esb1, 2 and 3, respectively.
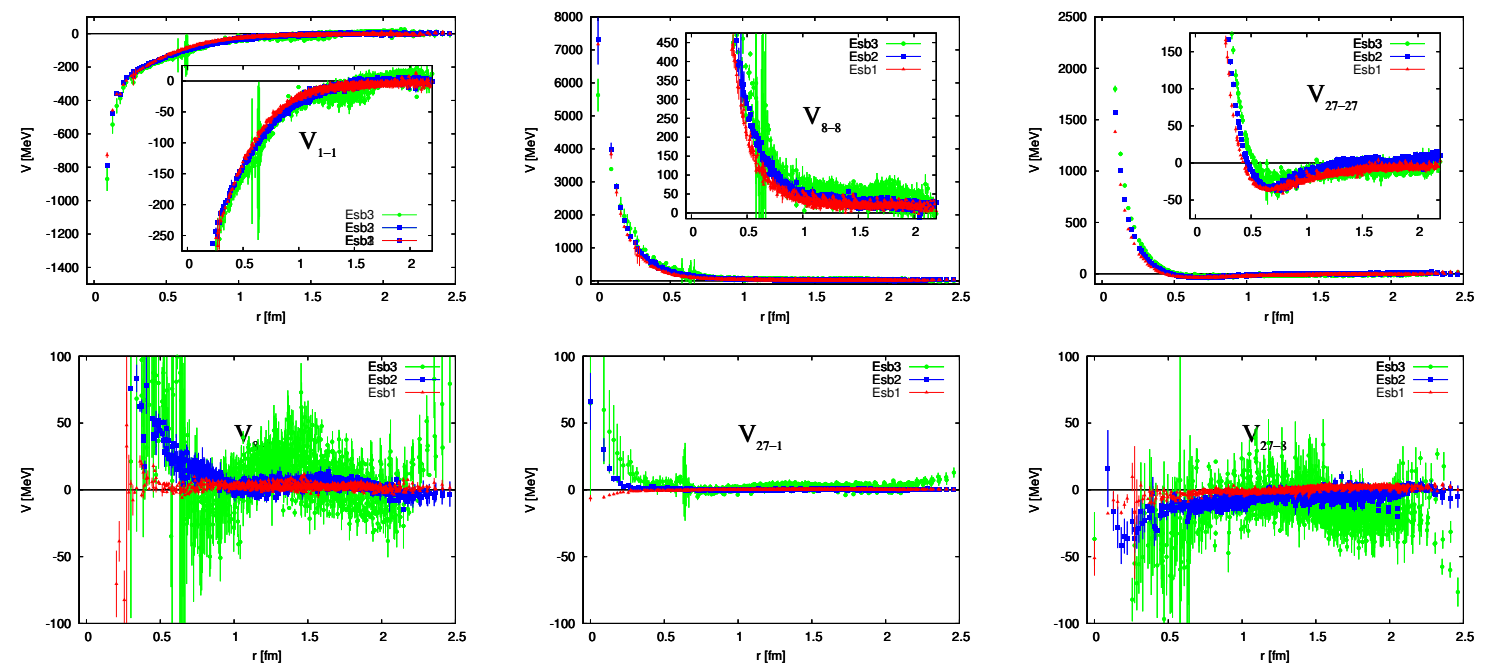

Figure 2: Potentials in ${ }^{1} S_{0}$ with $S=-2$ and $I=0$ in SU(3) irrep basis. Upper three panels are diagonal parts of potential matrix. Lower three panels are off-diagonal ones. Red, blue and green symbols stand for the result with Esb1, 2 and 3, respectively.

suppressed. This property could favor a formation and an observation of the $\Xi$ hypernuclei in experiments. Reduction of light flavored $u$ and $d$ quark masses leads to an enhancement of transition potentials especially in short distances.

The potentials are easily transformed from baryon basis to $\mathrm{SU}(3)$ irreducible representation (irrep) basis by using Clebsh-Gordan coefficients. It is instructive to see the potentials in irrep basis because they clearly show an essence of $B B$ interactions. Fig. 2 shows the potentials in ${ }^{1} S_{0}$ with $S=-2$ and $I=0$ in SU(3) irrep bases. As is expected from Ref. [4], we can see strongly attractive potential for flavor singlet state and repulsive potential for octet state. For both $V_{1-1}$ and 

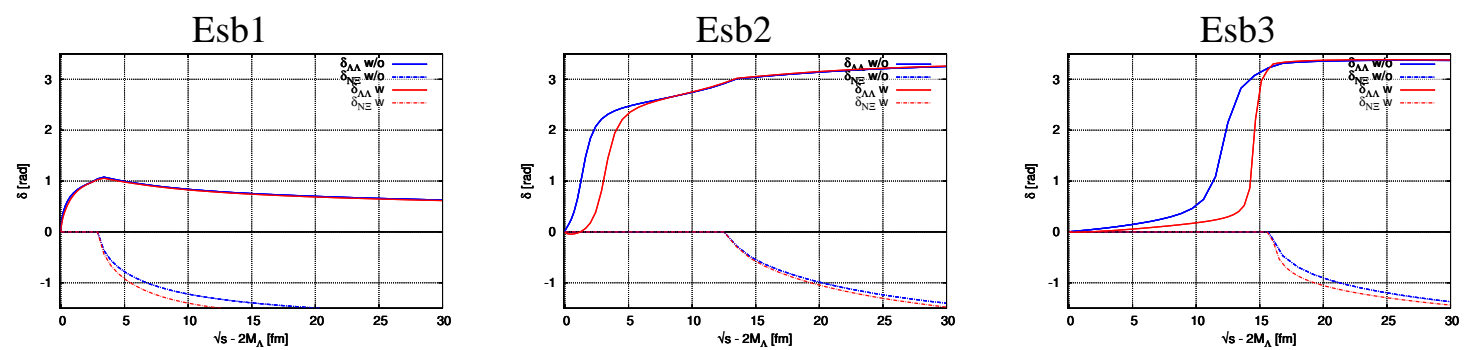

Figure 3: Preliminary results of $\Lambda \Lambda$ and $N \Xi$ phase shifts for Esb1, 2 and 3 respectively from left to right. Solid line stands for the $\delta_{\Lambda \Lambda}$ and Dotted line for the $\delta_{N \Xi}$. Blue color means the calculation turning off the offdiagonal parts of potential matrix in irrep basis. Red color indicates the full culculation in our formulation.

$V_{8-8}$ potentials, strength of them are gradually increased with decreasing quark masses. For the case of $V_{27-27}$, we can see the similar shape of potential to nuclear force. Short range parts of potentials are increased as the mass difference between $u d$ and $s$ quarks become larger. However we can not find clear enhancements at long distances of potentials.

Transition potentials between different irreps are presented in lower three panels in Fig. 2 and they could be an effective measure for the SU(3) breaking effects because it does not exist if the flavor symmetry is exact. As it is expected, they are slightly enhanced as increasing the quark mass difference between light and strange quark masses. This result tells us that the symmetry breaking effects are not so large compared to the diagonal potentials.

Using the potentials, the $\Lambda \Lambda$ and $N \Xi$ phase shifts are calculated, given in Fig. 3 In these figures, we performed two ways of calculations which are done with (w/) off-diagonal potentials in irrep basis and without (w/o) them. Comparing these two calculations, SU(3) breaking effects in $B B$ potential is not negligible but it shifts only a few $\mathrm{MeV}$ of the energy of H-dibaryon. In Fig. 3 , we can see the clear resonance shape in their phase shifts for Esb2 and 3. For Esb1, there is bound state below $\Lambda \Lambda$ threshold. Thus the binding energy of H-dibaryon from $N \Xi$ threshold is getting smaller as decreasing of quark masses, and finally it becomes resonance state as a result of going through the $\Lambda \Lambda$ threshold.

\section{Conclusions and Outlook}

We have investigated the $S=-2 B B$ potentials from $2+1$ flavor lattice QCD by considering the $\Lambda \Lambda, N \Xi$ and $\Sigma \Sigma$ coupled channels. In this work, we can confirm that HAL QCD method is successfully extend to the coupled channel systems [15] 16]. Combining the coupled channel formalism with the time-dependent Schrödinger type equation [16], we can get rid of ambiguities of potential. We have found that potentials in particle basis with the SU(3) breaking have similar properties to those of unitary rotated $B B$ potentials in $\mathrm{SU}(3)$ limit [4]. In our preliminary calculations, we found that the bound $\mathrm{H}$-dibaryon near SU(3) symmetry point tends to be a resonance as the $\mathrm{SU}(3)$ breaking is getting larger.

In future investigations, we will calculate for lighter quark masses towards the physical point with larger SU(3) breaking effects to trace the fate of H-dibaryon in the real world. 


\section{Acknowledgements}

We are grateful for authors and maintainers of CPS++ [19], whose modified version is used for our simulations. We also thank PACS-CS Collaboration and ILDG/JLDG [17] for providing gauge configurations. All numerical computation have been performed on the KEK supercomputer system-A. This work is supported by SPIRE (Strategic Program for Innovative Research), Grantin-Aid of the Ministry of Education, Science and Technology, Sports and Culture (Nos. 24740144, 23540321) and the Grant-in-Aid for Scientific Research on Innovative Areas (No. 2004:20105001, 20105003).

\section{References}

[1] N. Ishii, S. Aoki and T. Hatsuda, Phys. Rev. Lett. 99 (2007) 022001.

[2] S. Aoki, T. Hatsuda and N. Ishii, Prog. Theor. Phys. 123 (2010) 89.

[3] H. Nemura, N. Ishii, S. Aoki and T. Hatsuda, Phys. Lett. B673 (2009) 136.

[4] T. Inoue et al. [HAL QCD collaboration], Prog. Theor. Phys. 124 (2010) 591.

[5] Y. Ikeda [for HAL QCD Collaboration], PoS LATTICE 2011 (2011) 159.

[6] K. Murano, N. Ishii, S. Aoki and T. Hatsuda, Prog. Theor. Phys. 125 (2011) 1225.

[7] T. Doi et al. [HAL QCD Collaboration], Prog. Theor. Phys. 127 (2012) 723.

[8] S. Aoki et al. [HAL QCD Collaboration], Prog. Theor. Exp. Phys. (2012) 01A105.

[9] B. Charron et al. [HAL QCD Collaboration], in these proceedings.

[10] M. Oka, K. Shimizu and K. Yazaki, Prog. Theor. Phys. Suppl. 137 (2000) 1.

[11] T. Inoue et al. [HAL QCD Collaboration], Phys. Rev. Lett. 106 (2011) 162002.

[12] T. Inoue et al. [HAL QCD Collaboration], Nucl. Phys. A 881 (2012) 28.

[13] R. L. Jaffe, Phys. Rev. Lett. 38 (1977) 195 [Erratum-ibid. 38 (1977) 617].

[14] S. R. Beane et al. [NPLQCD Collaboration], Phys. Rev. Lett. 106 (2011) 162001.

[15] S. Aoki et al. [HAL QCD Collaboration], Proc. Jpn. Acad., Ser. B, 87 (2011) 509.

[16] N. Ishii et al. [HAL QCD Collaboration], Phys. Lett. B 712 (2012) 437.

[17] See "http://www.lqcd.org/ildg" / "http://www.jldg.org".

[18] S. Aoki et al. [PACS-CS Collaboration], Phys. Rev. D 79 (2009) 034503.

[19] Columbia Physics System(CPS), http://qcdoc.phys.columbia.edu/cps.html 